\title{
Is speeding more likely during weekend night hours? Evidence from sensor-collected data in Montreal
}

\author{
Shahram Heydari \\ (Corresponding Author) \\ Department of Civil, Maritime and Environmental Engineering \\ University of Southampton \\ Southampton, United Kingdom \\ s.heydari@soton.ac.uk

\section{Luis F. Miranda-Moreno} \\ Department of Civil Engineering and Applied Mechanics \\ McGill University \\ Montreal, Canada \\ luis.miranda-moreno@mcgill.ca

\section{Liping Fu} \\ Department of Civil and Environmental Engineering \\ University of Waterloo, \\ Waterloo, Canada \\ lfu@uwaterloo.ca
}

Word count: $3601+3$ (tables and figure) $* 250=4351$ 


\begin{abstract}
A number of traffic safety studies have investigated temporal variations in road safety indicators such as crash frequency, confirming that such variations exist. This paper examined whether speeding is more likely on weekend nights relative to all other times of the day by directly comparing speeding during weekends and weekdays. To this end, we analysed a sample of local streets, in Montreal, for which speed data were collected automatically using traffic analyser sensors. We found that, interestingly, weekend speeding was less likely to occur during night hours, whereas it was more likely to occur during evening and midday hours. Among other findings, the results indicated that one-way streets and those having a speed limit of $50 \mathrm{~km} / \mathrm{h}$ were slightly less prevalent in speeding on weekends. Our results can be useful in designing road safety interventions, including publicity campaigns and police enforcement, which aim at reducing speeding behaviours.
\end{abstract}

Keywords: Speeding behaviour, weekends, weekdays, speed limits, temporal variation

\title{
1. Introduction
}

Active travel modes safety is a major concern in many urban settings. For example, more than 15\% of Canadian road fatalities were pedestrians for the period 2013 to 2017, and most of pedestrian road fatalities occurred on urban roads (Transport Canada 2017). In this regard, vehicle operating speed and its traffic safety implications are of critical importance, especially in urban areas, due to the high prevalence of walking and cycling. Therefore, local authorities are often concerned about drivers' compliance with posted speed limits since car impact speed has a 
significant effect on injury severity levels sustained by pedestrians and cyclists (Hussain et al. 2019).

Some studies have examined the effect of reduced speed limits on speeding and safety (De Pauw et al. 2013; Heydari and Miranda-Moreno 2013; Islam et al. 2014; Heydari et al. 2014; Sun et al. 2018). These studies found that speed limit reduction is in general an effective safety intervention resulting in reduced operating speed, crash frequency, and severity. De Pauw et al. (2014), for instance, found that a $20 \mathrm{~km} / \mathrm{h}$ reduction in speed limits, from $90 \mathrm{~km} / \mathrm{h}$ to $70 \mathrm{~km} / \mathrm{h}$, resulted in a 5\% decrease in crash rates and a 33\% reduction in severe injuries and fatalities. In a similar study, Heydari et al. (2014) found that reducing speed limit from $50 \mathrm{~km} / \mathrm{h}$ to $40 \mathrm{~km} / \mathrm{h}$ was effective in reducing vehicle operating speed; however, this intervention did not reduce excessive speeding behaviours (speed $>80 \mathrm{~km} / \mathrm{h}$ ).

Given the importance of operating speed, a number of studies have focused on modelling vehicle operating speed (Dinh and Kubota 2013; Eluru et al. 2013). For example, Eluru et al. (2013) modelled operating speed as a function of roadway characteristics and temporal variables. A number of studies examined the effect of car impact speed on pedestrian fatality-injury risk (Anderson et al. 1997; Davis 2001; Rosén and Sander 2009; Kong and Yang 2010; Tay et al. 2011; Höskuldur 2015; Hussain et al. 2019). Based on a comprehensive study, for example, Rosén and Sander (2009) provided a risk function to explain the relationship between car impact speed and adult pedestrian fatalities. The authors found that the fatality risk at a car impact speed of $50 \mathrm{~km} / \mathrm{h}$ was two times higher than the fatality risk at $40 \mathrm{~km} / \mathrm{h}$. This risk was more than three times higher with respect to an impact speed of $30 \mathrm{~km} / \mathrm{h}$. Therefore, speed can be used as an indicator of traffic safety, especially in urban areas. A detailed literature review of research efforts investigating 
fatality-injury risk among pedestrians as a function of car impact speed can be found in Rosén et al. (2011) and Hussain et al. (2019).

Temporal variations have been shown to influence crash frequencies and severities. Traffic conditions and travel patterns are different between weekends and weekdays (Rakha and Van Aerde 1995), and driving during weekends is usually considered to be associated with a higher risk compared to weekdays (Farmer and Williams 2005; Blackman and Haworth 2013). For instance, Pai and Saleh (2008) and Gray et al. (2008) found that crashes occurring during weekends were more severe than those occurring during weekdays. Anowar et al. (2013) compared crashes between statutory holidays and regular weekends and found that injury fatal crashes were more prevalent during statutory holidays compared to weekends. Regardless of whether weekends impose a higher or lower risk to road users, almost all studies confirm differences caused by temporal variations (Christoforou et al. 2010; Rifaat et al. 2011).

The majority of previous studies have explored the effect of temporal factors on crash frequencies or injury severities. However, very limited number of studies have investigated how weekends and weekdays differ in terms of speeding incidences, and when related issues are discussed, the focus often is not on comparing speeding during weekends and weekdays. In this regard, for example, Giles (2004), using a data set from Western Australia for the years 1999 to 2000, found that operating speed was slightly lower on weekends relative to weekdays. Familar et al. (2011) found that speeding is more likely on weekend nights than weekday nights. These studies use weekends and weekdays as explanatory variables in their statistical analysis. However, a direct comparison would provide more detailed insights. To this end, the objective of our paper is to directly compare speeding between weekends and weekdays, examining how the effects of various 
road characteristics and time variables vary between weekends and weekdays. Specifically, with respect to compliance with posted speed limits, we investigate whether weekend nights are riskier than all other times of the day, including weekday nights.

\section{Data}

A sample of 54 randomly-selected local urban streets in Montreal was used in this paper. The data set was collected automatically, using traffic analyser sensors installed on the road pavement, during an important data collection campaign following the Montreal transportation plan in 2008. This plan was partly designed to study operating speed and its determinants on local streets in Montreal. Sites were selected randomly and included local streets with posted speed limits of 50 $\mathrm{km} / \mathrm{h}$ and $40 \mathrm{~km} / \mathrm{h}$. In the site selection process, different criteria were considered to ensure drivers would reach their maximum speed without being influenced by major external factors. For example, sites with bicycle or bus lane, those in the proximity of schools and parks having a posted speed limit of $30 \mathrm{~km} / \mathrm{h}$, and sites with calming measures were not considered. In addition, the length of the selected sites was decided to be at least 200 meters to allow drivers to reach a relatively high speed. Experts have also visited the selected sites in order to ensure the validity of the sample.

The operating speed of vehicles was measured through a fully automated system during a five-day period (Thursday, Friday, Saturday, Sunday, and Monday) in October 2009. Note that the measurements correspond to traffic flow speed, not free flow speed. Speed measurements were directly provided by data collection sensors in the form of speed categories (e.g., 0-30 km/h, 30$40 \mathrm{~km} / \mathrm{h}$, etc.); therefore, the hourly number of vehicles related to each speed category was made available. Since we were interested in identifying the delineation of explanatory factors between 
weekends and weekdays in terms of speeding behaviours, we obtained the overall hourly number of speeding incidences during both weekend and weekday periods. Speed data were collected at each site during a five-day period $(24 * 5=120$ hours). For example, in the first hour (12 am to 1 am) after aggregating data from multiple days, there were 68 speeding incidences out of which 28 occurred during weekend. Besides speeding incidences, several temporal variables and roadway characteristics were considered as described below. Descriptive statistics are reported in Table 1.

After removing missing measurements, our final data included 1,164 observations (hours), recording 82,901 vehicles exceeding posted speed limits in total, among which 32,641 (i.e., 39.37\%) speeding incidences occurred during the weekend. With respect to total traffic volume passing through data collection sensors, $37 \%$ and $38.5 \%$ of vehicles exceeded posted speed limits during weekends and weekdays, respectively. The data were collected on local streets with posted speed limits of $40 \mathrm{~km} / \mathrm{h}$ and $50 \mathrm{~km} / \mathrm{h}$. Thus, a categorical variable for speed limit was introduced in the model to account for likely variations in speeding behaviours with respect to the aforementioned speed limits. Accounting for different speed limits is justifiable as drivers' compliance and attitude toward different speed limits may vary. In our sample, 28 sites had a speed limit of $50 \mathrm{~km} / \mathrm{h}$ and the remaining sites had a speed limit of $40 \mathrm{~km} / \mathrm{h}$.

The presence of horizontal curves, whether the street was one-way, and time variables (evening, night, and midday) were also used in the form of categorical variables. Finally, the number of sidewalks (a roadway can have a sidewalk on one side, two sides or neither of the sides), as an indicator of pedestrian activity, was taken into account. In our sample, $12 \%$ of the sites had a sidewalk on one side only, $66 \%$ had sidewalks on both sides, and the remaining $22 \%$ had no sidewalk. Since the data were collected automatically using traffic sensors, information on drivers’ 
demographics (age, sex, experience, etc.) or conditions (e.g., drink-driving) was not available. Note that this study does not investigate possible causes of speeding, but only the variation of factors between weekend speeding and weekdays speeding.

\section{Statistical analysis}

Given the structure of the data explained above, a binomial regression model was used to achieve the objective of the paper. The focus was to compare speeding incidences during weekend and weekdays, using a series of contributing factors such as time variables and roadway characteristics. In other words, the idea was to investigate factors that delineate between weekend speeding and weekday speeding incidences. Therefore, we were mainly interested in interpreting regression coefficients. The logit link function, which is convenient in terms of interpretation, was selected. Note that the logit model is used in the crash literature to investigate temporal variations (see, e.g. Anowar et al. 2013).

Note that the data set used in this paper is in the form of panel data in which each site comprises multiple measurements collected during different hours of observations. For panel data, usually a random effects model is used. However, we discuss our findings based on a fixed effects model because of the following reasons. First, the variance of the random effects was found to be very small (i.e., $0.014[0.007,0.026]$ ), thus being negligible. Second, the main focus of this study is on the interpretation of the odds ratios and their general increasing or decreasing effect on the outcome of interest, speeding during weekends versus weekdays. Also, note that in a fixed effects logistic regression, the interpretation of odds ratios is straightforward, whereas odds ratios in random effects logistic models are given the random effects. 
Suppose $\mathrm{j}$ denotes hourly observations and $\mathrm{r}_{\mathrm{j}}$ denotes the number of vehicles exceeding posted speed limits (i.e., speeding incidences) in each hour during weekend among the total number of speeding incidences $\mathrm{v}_{\mathrm{j}}$ that occur in each hour during both weekend and weekday periods. Given the probability $\mathrm{p}_{\mathrm{j}}$ of speeding incidences occurring during a weekend, the binomial regression model can be defined as in (1). The aim is to model $\mathrm{p}_{\mathrm{j}}$ as a function of explanatory variables $X_{j}$, with a corresponding vector of regression coefficients $\alpha=\left(\alpha_{0}, \alpha_{1}, \ldots, \alpha_{\mathrm{m}}\right)$.

$$
\begin{aligned}
& \mathrm{r}_{\mathrm{j}} \mid \mathrm{p}_{\mathrm{j}} \sim \operatorname{Binomial}\left(\mathrm{p}_{\mathrm{j}}, \mathrm{v}_{\mathrm{j}}\right) \\
& \operatorname{logit}\left(\mathrm{p}_{\mathrm{j}}\right)=\log \left(\mathrm{p}_{\mathrm{j}} /\left(1-\mathrm{p}_{\mathrm{j}}\right)\right)=\alpha_{0}+\alpha_{1} \mathrm{x}_{\mathrm{j} 1}+\cdots+\alpha_{\mathrm{m}} \mathrm{x}_{\mathrm{jm}}
\end{aligned}
$$

\section{Results and Discussion}

The results (regression coefficient estimates and their associated odds ratios) are reported in Table 2. We used Stata 13 software (StataCorp, 2013) for analysing the data. The results indicate that in general roadway characteristics do not have a strong impact on weekend speeding. In other words, the effects of roadway characteristics delineate slightly between speeding incidences that occur during weekend and those occurring during weekdays. On the other hand, there is a relatively large variation in terms of time variables. The major difference can be seen between night time hours (12:00 am - 6:00 am) and all other times of the day. Travel patterns are often different during weekends and weekdays. In fact, most weekend trips are of discretionary nature, involving a higher rate of drunk-driving (Anowar et al. 2013). Therefore, temporal variations in speeding are expected.

It can be implied from the estimated odds ratios that, as the number of sidewalks increases, the likelihood of speeding during weekend increases marginally. Specifically, for one unit increase 
in the number of sidewalks, the odds of speeding during weekends increases by a factor of 1.07 ; i.e. $7 \%$. As lane width increases, the likelihood of speeding during weekends decreases. Note that this does not imply causality; instead, some sort of hidden association could play a role here. In effect, this finding might be an indication of the fact that perhaps travel patterns and/or other factors, which are similar among sites with wider lanes, reduce the likelihood of speeding during weekends. To draw conclusive conclusions an in-depth study would be necessary. We also found that speeding incidences were less likely to occur on one-way streets, on streets where the speed limit was $50 \mathrm{~km} / \mathrm{h}$, and on streets with at least one horizontal curve. For example, the odds of speeding on weekends is on average $6 \%(100 *(0.94-1))$ lower among sites with a speed limit of $50 \mathrm{~km} / \mathrm{h}$ compared to sites with a speed limit of $40 \mathrm{~km} / \mathrm{h}$.

With respect to time variables, speeding behaviours on weekends were less likely to occur during night hours compared to evening or midday hours. Specifically, weekend speeding was on average $22.5 \%((0.775-1) * 100)$ lower during night hours compared to rush hours, the reference category. We can also infer from the results that the likelihood of speeding is lower during weekend nights relative to weekday nights, and it is the lowest during weekend night times compared to all other time periods in weekend and weekdays. This contradicts the general belief that night hours during weekends are more risky in terms of speeding, for example, due to the fact that driving under the influence of drugs/alcohol and reckless driving behaviours are expected to be more prevalent. Further in-depth investigation is needed to understand the reason(s) behind this finding. One possible explanation is that, for instance, drivers who drink may travel at lower speeds during weekend night hours to avoid being caught by the police since police enforcement is often more frequent during weekend nights. 
One limitation of our data was that drivers’ information (e.g., drunk-driving, age, etc.) was not available since data were collected automatically using traffic analyser sensors. Had such data been available, we would have been able to undertake a more comprehensive analysis providing detailed insights on explanatory factors associated with speeding during weekends.

\section{Conclusions}

Pedestrians and cyclists are extremely vulnerable to car impact speed. Therefore, in urban areas such as Montreal, where walking and cycling are frequent, speeding is of a major concern. To this end, municipalities aim to make urban streets as risk-free as possible. Time variables (e.g., days of the week, hours of the day, etc.) often affect road safety indicators such as crash frequency and speeding, and weekends or holiday periods are usually considered to be more dangerous than weekdays. In accordance with previous research, our study confirms that temporal variations (e.g., weekend versus weekday) affect traffic safety. While most previous studies have investigated the effect of temporal variations on crash frequencies and/or injury severity propensities, we examined how such variations influence speeding behaviours by directly comparing factors that delineate between speeding incidences during weekends and weekdays. Specifically, relying on data collected automatically using traffic analyser sensors, this paper examined whether weekend night hours pose a higher risk to road users compared to all other times of the day in terms of speeding behaviours.

We found that speeding on weekends was more likely to occur during evening and midday hours whereas it was less likely to occur during night hours. Interestingly, the results also imply that the odds of speeding are lower during weekend nights compared to weekday nights. The likelihood of speeding was slightly lower on one-way streets and those having a speed limit of 50 
$\mathrm{km} / \mathrm{h}$. Note that the assumption here is that the one-week period observed is representative of the other weeks. Hence, a more conclusive conclusion can be reached by conducting a sensitivity analysis considering other data collection periods across the year.

In terms of practical implications, knowing where and when speeding risk is higher could be useful in designing safety improvement programs, including engineering interventions, planning enforcement, and designing publicity campaigns with the aim of raising road users' awareness about speeding. In fact, our results can help decision makers in reviewing current traffic safety interventions, including traffic enforcement strategies. Considering the case study analysed in this paper, for example, we found that speeding during night hours were over-represented on weekdays; therefore, to be more effective, police enforcement programs should consider the fact that night hours speeding during weekdays are more likely than night hours speeding during weekends. The results also showed that evening and midday speeding were over-represented on weekends. Thus, traffic enforcement strategies should be programmed to reflect such temporal variations on weekends.

Relative to geometry of the sites, speeding on one-way streets was found to be less likely during weekends. Therefore, decision makers might not want to select one-way streets (over twoway streets) for traffic enforcement activities on weekends, provided that both types of streets have similar traffic volumes. Similarly, urban streets with a posted speed limit of $50 \mathrm{~km} / \mathrm{h}$ should not be of a major concern during weekends since these streets were found to be less prevalent with respect to speeding on weekends compared to weekdays. Another useful practical implication relates, for example, to publicity campaigns. In fact, our results can help decision makers to plan publicity campaigns to provide an effective strategy towards promoting behavioural change with 
respect to speeding. This can raise road users' awareness regarding where and when the likelihood of speeding is higher than usual.

\section{ACKNOWLEDGEMENT}

The authors would like to thank the Montreal Department of Transportation for providing the data.

\section{REFERENCES}

Anderson, R.W.G., McLean, A.J., Farmer, M.J.B., Lee, B.H., and Brooks, C.G. 1997. Vehicle travel speeds and the incidence of fatal pedestrian crashes. Accident Analysis and Prevention, 29(5): 667-674.

Anowar, S., Yasmin, S., and Tay, R. 2013. Comparison of crashes during public holidays and regular weekends. Accident Analysis and Prevention, 51: 93-97.

Blackman, R.A., and Haworth, N.L. 2013. Comparison of moped, scooter and motorcycle crash risk and crash severity. Accident Analysis and Prevention, 57: 1-9.

Christoforou, Z., Cohen, S., and Karlaftis, M. 2010. Vehicle occupant injury severity on highways: an empirical investigation. Accident Analysis and Prevention, 42(6): 1606-1620.

Davis, G.A. 2001. Relating severity of pedestrian injury to impact speed in vehicle pedestrian crashes. Transportation Research Record, 1773: 108-113.

De Pauw, E., Daniels, S., Thierie, M., and Brijs, T. 2014. Safety effects of reducing the speed limit from $90 \mathrm{~km} / \mathrm{h}$ to $70 \mathrm{~km} / \mathrm{h}$. Accident Analysis and Prevention, 73: 252-261.

Dinh, D., and Kubota, H. 2013. Drivers' perceptions regarding speeding and driving on urban residential streets with a 30km/h speed limit. IATSS Research, 37(1): 30-38.

Eluru, N., Chakour, V., Chamberlain, M., and Miranda-Moreno L. 2013. Modeling vehicle operating speed on urban roads in Montreal: a panel mixed ordered probit fractional split model. Accident Analysis and Prevention, 59: 125-134.

Familar, R., Greaves, S., and Ellison, A. 2011. Analysis of Speeding Behavior: Multilevel Modeling Approach. Transportation Research Record, 2237(1): 67-77.

Farmer, C., and Williams, A. 2005. Temporal factors in motor vehicle crash deaths. Injury Prevention, 11(1): 18-23. 
Giles, M.J. 2004. Driver speed compliance in Western Australia: a multivariate analysis. Transport Policy, 11(3): 227-235.

Gray, R., Quddus, M., and Evans, A. 2008. Injury severity analysis of accidents involving young male drivers in Great Britain. Journal of Safety Research, 39(5): 483-495.

Heydari, S., and Miranda-Moreno, L.F. 2013. A mixed-effect statistical model for before-after speed studies. Proceedings, Annual Conference - Canadian Society for Civil Engineering, 4(January): 3233-3242.

Heydari, S., Miranda-Moreno, L.F., and Fu, L. 2014. Speed limit reduction in urban areas: a before-after study using Bayesian generalized mixed linear models. Accident Analysis and Prevention, 73: 252-261.

Höskuldur K. 2015. Is 30km/h a 'safe' speed? Injury severity of pedestrians struck by a vehicle and the relation to travel speed and age. IATSS Research, 39(1): 42-50.

Hussain, Q., Feng, H., Grzebieta, R., Brijs, T., and Olivier, J. 2019. The relationship between impact speed and the probability of pedestrian fatality during a vehicle-pedestrian crash: a systematic review and meta-analysis. Accident Analysis and Prevention, 129: 241-249.

Islam, T. Md., El-Basyouny, K., and Ibrahim, S.E. 2014. The impact of lowered residential speed limits on vehicle speed behavior. Safety Science, 62: 483-494.

Kong, C., and Yang, J. 2010. Logistic regression analysis of pedestrian casualty risk in passenger vehicle collisions in China. Accident Analysis and Prevention, 42(4): 987-993.

Li, G., Lyons, M., Wang, B., Yang, J., Otte, D., and Simms, C. 2017. The influence of passenger car front shape on pedestrian injury risk observed from German in-depth accident data. Accident Analysis and Prevention, 101: 11-21.

Pai, C-H., and Saleh, W. 2008. Exploring motorcyclist injury severity in approach-turn collisions at T-junctions: focusing on the effects of driver's failure to yield and junction control measures. Accident Analysis and Prevention, 40(2): 479-486.

Rakha, H., and Van Aerde, M. 1995. Statistical analysis of day-to-day variations in real-time traffic flow data. Transportation Research record, 1510: 26-34.

Rifaat, S., Tay, R., and de Barros, A. 2011. Effect of street pattern on the severity of crashes involving vulnerable road users. Accident Analysis and Prevention, 43(1): 276-283. 
Rosén, E., and Sander, U. 2009. Pedestrian fatality risk as a function of car impact speed. Accident Analysis and Prevention, 41(3): 536-542.

Rosén, E., Stigson, H., and Sander, U. 2011. Literature review of pedestrian fatality risk as a function of car impact speed. Accident Analysis and Prevention, 43(1): 25-33.

StataCorp. 2013. Stata Statistical Software: Release 13. College Station, TX: StataCorp LP.

Sun, D., El-Basyouny, K., Ibrahim, S., and Kim, A. 2018. Are school zones effective in reducing speeds and improving safety? Canadian Journal of Civil Engineering, 45(12): 1084-1092.

Tay, R., Choi, J., Kattan, L., and Khan, A. 2011. A multinomial logit model of pedestrian-vehicle crash severity. International Journal of Sustainable Transportation, 5(4): 233-249.

Transport Canada, 2017. https://www.tc.gc.ca/eng/motorvehiclesafety/resources-researchstatsmenu-847.htm 
TABLE 1 Descriptive Statistics of Data

\begin{tabular}{|c|c|c|c|c|}
\hline Variable & Frequency & Percent & & \\
\hline Night hours (12 AM to 6 AM) & 258 & 22.16 & - & - \\
\hline Midday hours (10 AM to 3 PM) & 321 & 27.58 & - & - \\
\hline Evening hours (7 PM to $12 \mathrm{AM}$ ) & 266 & 22.85 & - & - \\
\hline Rush hours (6 AM to $10 \mathrm{AM}, 3 \mathrm{PM}$ to $7 \mathrm{PM}$ ) & 319 & 27.40 & - & - \\
\hline Speed limit: 1 if $50 \mathrm{~km} / \mathrm{h}, 0$ otherwise & 612 & 52.58 & - & - \\
\hline One-way ( 1 if one way street, 0 otherwise) & 399 & 34.28 & - & - \\
\hline \multirow[t]{2}{*}{ Horizontal curve ( 1 if horizontal curve is present, 0 otherwise) } & 137 & 11.77 & - & - \\
\hline & Mean & Std. Dev. & Min & Max \\
\hline Lane width (meters) & 5.09 & 1.26 & 3 & 9.25 \\
\hline Sidewalk (number of sidewalks) & 1.43 & 0.83 & 0 & 2 \\
\hline N (total hourly traffic volume exceeding speed limit) & 71.22 & 118.26 & 1 & 892 \\
\hline Y (hourly traffic volume exceeding speed limit on weekend) & 28.04 & 43.46 & 0 & 240 \\
\hline
\end{tabular}

Note: The reference group for Midday, Evening, and Night hours is rush hours.

TABLE 2 Analysis Results

\begin{tabular}{|c|c|c|c|c|c|c|c|}
\hline \multirow{3}{*}{$\begin{array}{l}\text { Variable } \\
\text { Night hours }\end{array}$} & \multicolumn{4}{|c|}{ Model coefficient estimates } & \multicolumn{3}{|c|}{ Odds ratio estimates } \\
\hline & \multirow{2}{*}{$\begin{array}{l}\text { Mean } \\
-0.255\end{array}$} & \multirow{2}{*}{$\frac{\text { Std. Err. }}{0.025}$} & \multicolumn{2}{|c|}{$95 \%$ CI } & \multirow{2}{*}{$\begin{array}{l}\text { Mean } \\
0.775\end{array}$} & \multirow{2}{*}{$\begin{array}{c}\text { z-statistic } \\
-10.150\end{array}$} & \multirow{2}{*}{$\begin{array}{c}\mathrm{p} \text {-value } \\
0.000\end{array}$} \\
\hline & & & -0.305 & -0.206 & & & \\
\hline Evening hours & 0.080 & 0.021 & 0.038 & 0.121 & 1.083 & 3.790 & 0.000 \\
\hline Midday hours ${ }^{1}$ & 0.031 & 0.018 & 0.001 & 0.060 & 1.031 & 1.730 & 0.084 \\
\hline One-way & -0.052 & 0.019 & -0.090 & -0.014 & 0.949 & -2.690 & 0.007 \\
\hline Horizontal curve & -0.076 & 0.019 & -0.114 & -0.037 & 0.927 & -3.880 & 0.000 \\
\hline Sidewalks & 0.068 & 0.017 & 0.035 & 0.101 & 1.070 & 4.050 & 0.000 \\
\hline Speed limit & -0.062 & 0.016 & -0.094 & -0.030 & 0.940 & -3.820 & 0.000 \\
\hline Lane width & -0.013 & 0.006 & -0.024 & -0.002 & 0.987 & -2.230 & 0.026 \\
\hline Constant & -0.385 & 0.035 & -0.454 & -0.317 & - & -11.010 & 0.000 \\
\hline
\end{tabular}

\footnotetext{
${ }^{1}$ Significant at a $90 \%$ level of confidence
} 